\title{
Design and Implementation of Security Systems for Smart Home based on GSM technology
}

\author{
Jayashri Bangali $^{1}$ and Arvind Shaligram ${ }^{2}$ \\ ${ }^{1}$ Kaveri College of Science and Commerce \\ ${ }^{2}$ Department of Electronic Science \\ jayashri789@yahoo.com
}

\begin{abstract}
Security has becoming an important issue everywhere. Home security is becoming necessary nowadays as the possibilities of intrusion are increasing day by day. Safety from theft, leaking of raw gas and fire are the most important requirements of home security system for people. A traditional home security system gives the signals in terms of alarm. However, the GSM (Global System for Mobile communications) based security systems provides enhanced security as whenever a signal from sensor occurs, a text message is sent to a desired number to take necessary actions.

This paper suggests two methods for home security system. The first system uses web camera. Whenever there is a motion in front of the camera, it gives security alert in terms of sound and a mail is delivered to the owner. The second method sends SMS which uses GSMGPS Module (sim548c) and Atmega644p microcontroller, sensors, relays and buzzers.
\end{abstract}

Keywords: GSM (Global System for Mobile communications), Microcontroller, SMS (Short Message Service)

\section{Introduction}

Smart Home can be also known as Automated Home or intelligent home which indicates the automation of daily tasks with electrical appliances used in homes. This could be the control of lights, fans, viewing of the house interiors for surveillance purposes or giving the alarm alteration or indication in case of gas leakage.

Home security has changed a lot from the last century and will be changing in coming years [1]. Security is an important aspect or feature in the smart home applications [2]. The new and emerging concept of smart homes offers a comfortable, convenient, and safe environment for occupants. Conventional security systems keep homeowners, and their property, safe from intruders by giving the indication in terms of alarm. However, a smart home security system offers many more benefits.

This paper mainly focuses on the security of a home when the user is away from the place. Two systems are proposed, one is based on GSM technology and other uses web camera to detect the intruder.

The first security system uses a web camera, installed in house premises, which is operated by software installed on the PC and it uses Internet for communication. The camera detects motion of any intruder in front of the camera dimensions or camera range. The software communicates to the intended user via Internet network and at the same time it gives sound alert.

The second security system is SMS based and uses GSM technology to send the SMS to the owner. The proposed system is aimed at the security of Home against Intruders and Fire. 
In any of the above cases happens while the owners are out of their home then the device sends SMS to the emergency number which is provided to the system. The system is made up of three components: sensors, GSM-GPS Module (sim548c), Atmega644p microcontroller, relays to control the device and buzzers to give security alert signal in terms of sound.

\section{Market Survey}

According to the market research, the common parameters or characteristics of home security system are 24 hours monitoring of the intruder, ease of use, reliability, efficient, fast and precise notification system. Today numbers of home security systems are available in market.

In paper [3], a design which contains a home network including a GPRS/GSM gateway and three kinds of wireless security sensor nodes is presented. This system has a user interface and it can respond quickly to alarm incidents. In paper [4], a new method of moving object detection by combination of pixel illumination with its chroma in YUV color space is implemented. The algorithm of maintenance with 3 key values is discussed in this paper. In case of swaying objects, it is very robust and effective way of false alarms. Paper [5] discuses the detection and description based on an object oriented, statistical multi feature analysis of video sequences.

The system described in [6] monitors everything by moving cameras. The system can increase the efficiency of monitoring and can eliminate the blind spots of fixed cameras. In this system, a mobile manipulator is developed which is equipped with cameras at the arm end for purpose of monitoring. The system is based on SMS technology using any GSM modem/mobile is presented in [7]. The proposed remote control system works from anywhere in the world.

A low cost Short Message System (SMS) based home security system equipped with motion sensor, smoke detector, temperature sensor, humidity sensor and light sensors has been studied in [8]. The sensors are controlled by a microprocessor PIC 18F4520 through the SMS having password.

\section{Proposed System}

A model of smart home is prepared using low cost materials like acrylic, plywood etc., having sufficient strength as shown in Figure 1 to test the prototype of developed system. It consists of various sensors like IR, temperature sensor, gas sensors, light sensor etc. Home appliances like LED lights and fans are also connected to make the home energy efficient.

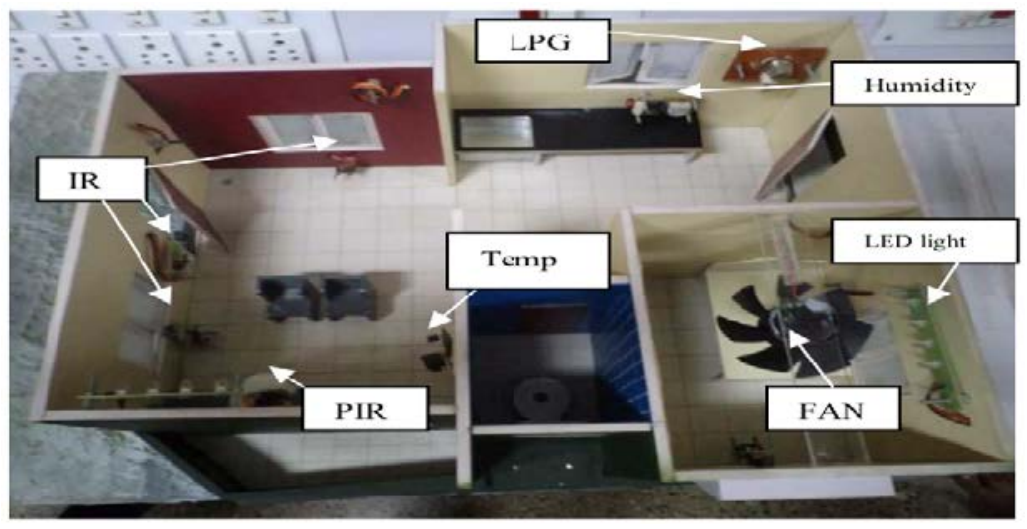

Figure 1. Model of Smart Home 
The proposed system is controlled by an Atmega644p microcontroller. It collects information from the sensors, makes a decision and sends SMS to a corresponding number by using a GSM modem. If it finds any interruption in its sensors (for example IR sensor) then microcontroller will send a SMS to the home owner. In the same way if the temperature is increased above certain point or gas sensor sensors is ON, a SMS will be sent to the home owner 'Fire at home' giving the indication of fire. The LDR (Light Dependent Resistor) is used to sense the light in a room and accordingly lights will be turn ON or OFF.

\section{Hardware Design}

Hardware of the system contains sensors, Atmega644p microcontroller, sim548c (GSM module), Buzzer, in system programmer and relays to control the appliances. The system design is shown in Figure 2.

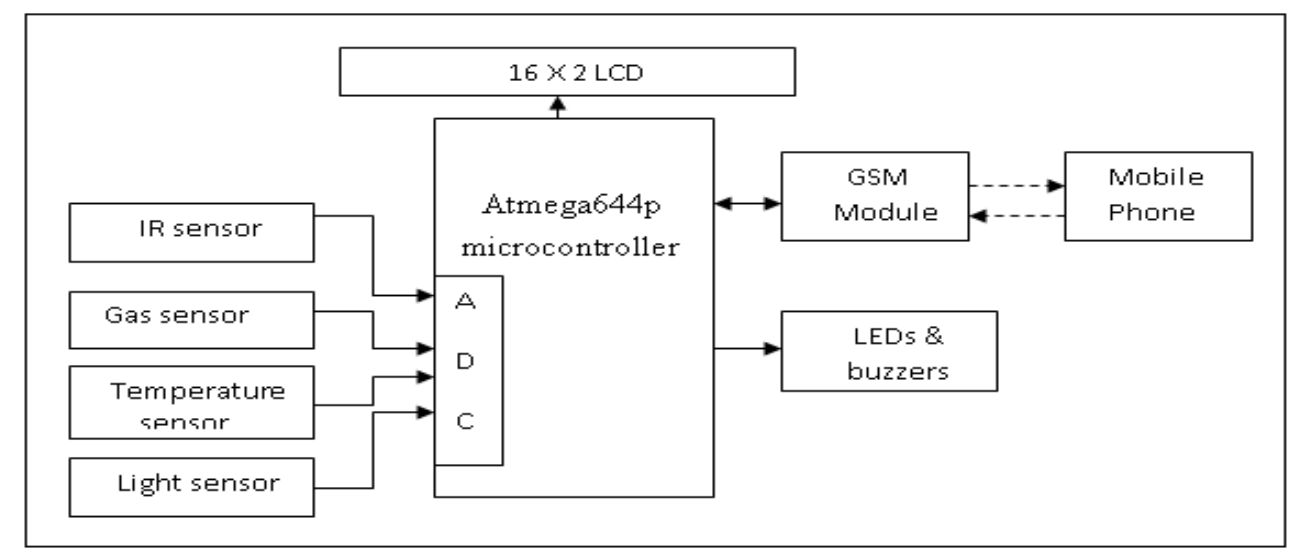

Figure 2. Proposed System Design

The outputs of all the sensors are connected to ADC. One IR is connected at window and other is at door. The entry from the window is treated as unauthorized entry and entry from door is treated as authorized entry. If there is authorized entry inside the home, lights will be turn ON after checking the light intensity in a room and for unauthorized entry buzzer will be turned ON. Temperature is continuously monitored, if it is high (greater than 45 degree) in case of fire, a SMS is sent ("Fire at home") to the home owner. If gas sensor is ON indicating the gas leakage then SMS will be send to the owner ('Gas Leakage').

\subsection{Microcontroller Unit}

The control module is built with the microcontroller IC. The central controller is Atmega644p which is 8-bit Microcontroller with 16/32/64K Bytes and in-System Programmable Flash. It is having advanced RISC architecture. It consists of Two 8-bit Timer/Counters with separate prescalers and Compare Modes, one 16-bit Timer/Counter with separate prescaler, compare Mode, and capture Mode, Real time counter with separate oscillator, six PWM channels, 8-channel 10-bit ADC and 32 Programmable I/O Lines.

\subsection{GSM Module Unit}

A SIM548C based quad band GSM module which supports GPS technology for satellite navigation is used. It provides GPRS multi-slot class10 / class8 capabilities and supports GPRS coding schemes CS-1, CS-2, CS-3, and CS-4. This module takes care of all your GSM- 
GPRS based communication requirements as well as provides live GPS data. There is an attached FT232RL USB interface for serial communication with PC or other serial devices. This USB port is also interfaced with the microcontroller. When connected to a PC, the port presents itself as a virtual serial (RS232) port. An HD44780U based LCD is embedded onboard (operates in 4-bit mode) interfaced with the microcontroller. A 10-pin programming interface is used to transfer (flash) the programs (in form of .HEX files) to the microcontroller. Another 10 pin header is attached to serves as the interface to external input sources (sensors) or output devices (LEDs).

AT Command is a set of commands or instructions which can be used to communicate (talk) with a GSM modem/mobile phone. AT commands are used to automatically receive the call on system from the preconfigured number and system also sends the message to preconfigured number about the intrusion indication through AT commands [9]. The AT commands of GSM-GPRS modules are given below.

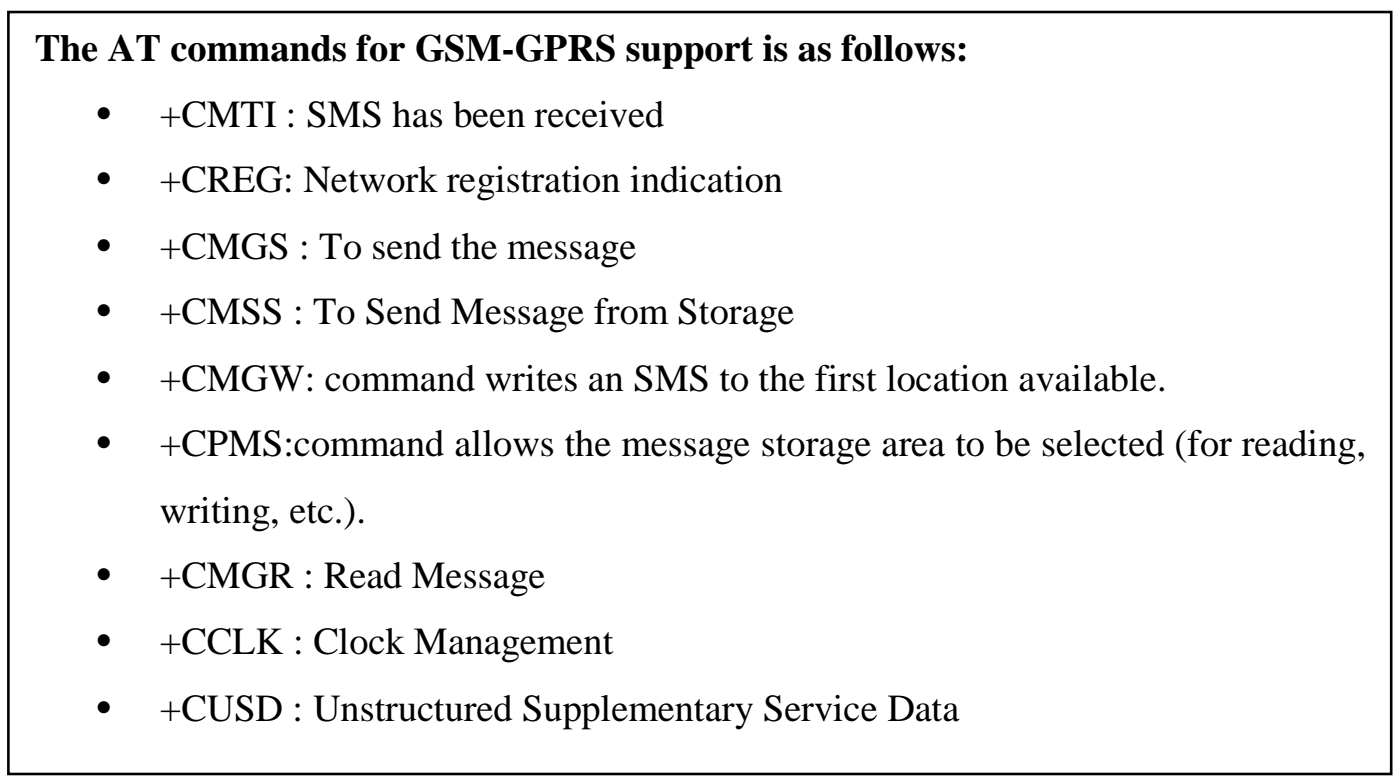

\subsection{Sensors used in the System}

Infrared (IR) sensors are used to detect the intruder. They are used at doors and at windows. The IR pair that is IR transmitter and IR receiver detects the obstacle within the range of 5-6 feet. The LM35 is used as temperature sensor whose output voltage is linearly proportional to the Celsius (Centigrade) temperature. It gives linear output $10.0 \mathrm{mV} /{ }^{0} \mathrm{C}$ as scale factor. Light Dependent Resistor (LDR) is used as a light sensor to sense the light intensity in the room. LDR gives the output voltage corresponding to the light intensity.

\section{Software Design}

The proposed system uses AVR microcontroller, programming is done in ' $\mathrm{C}$ ' language and to download the program into AVR chip, eXtreme burner is used.

\subsection{MikroC PRO and eXtreme Burner for AVR”}

In this system MikroC PRO is used to develop the program for AVR microcontroller. To get the HEX file from developed C program, eXtreme Burner software is used. The flow chart of whole system is shown in Figure 3. 


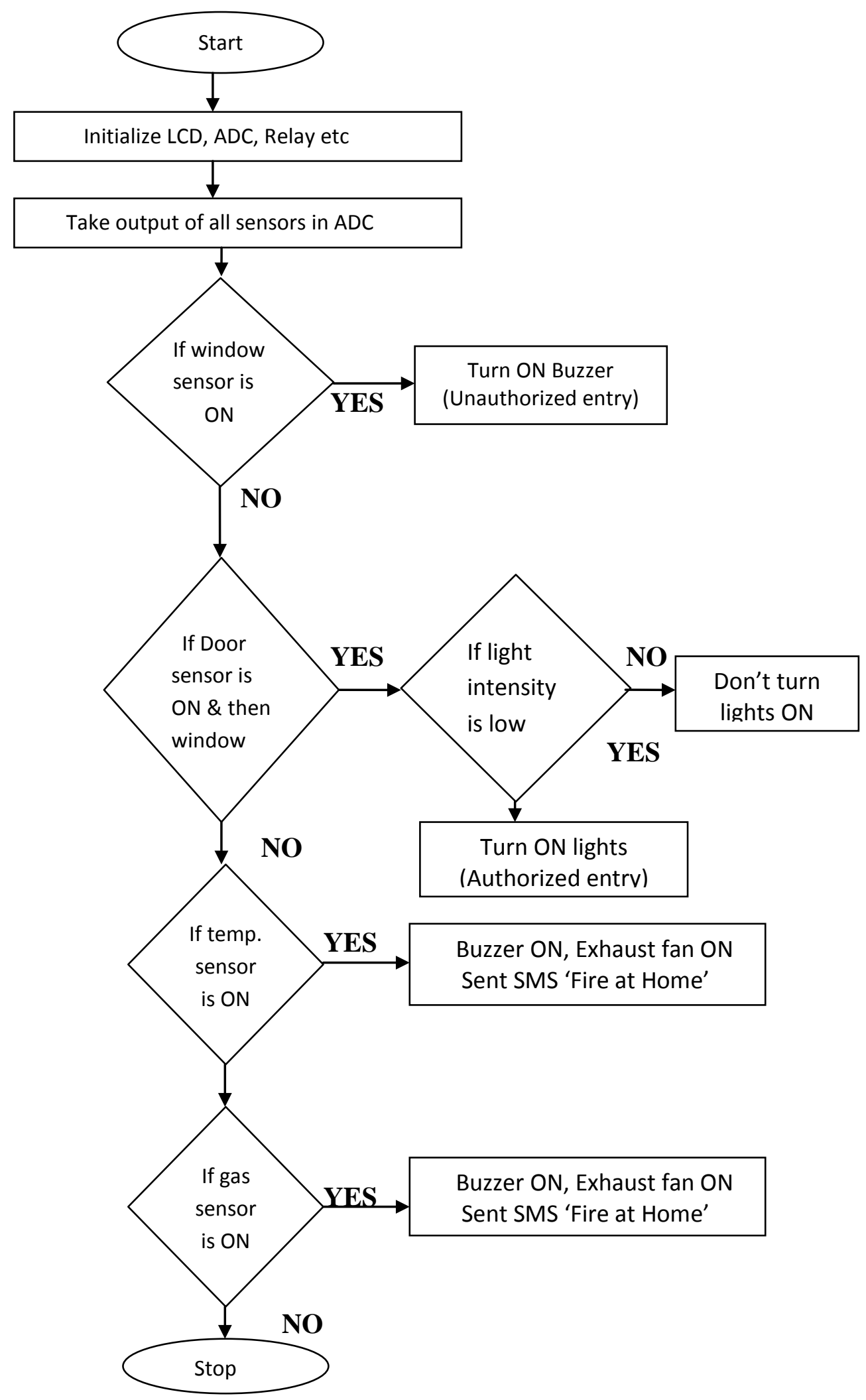

Figure 3. Flow Chart of the System 


\section{Security System using Web Camera}

The security system uses the Yawcam (Yet Another WebCAM Software) software to interface the web camera. Yawcam is Multilingual Java software for viewing and transferring images or videos from a webcam. Yawcam is a versatile tool for web camera utilization. With yawcam it is possible to observe the web camera, take snapshots or stream the video to the internet. Snapshots or video can be uploaded to the web using your own PC as a server. A mail can be sent by this software to the owner along with the images. The sensitivity and tolerance of images can be adjusted.

Yawcam is programmed in Java, but requires Windows 2000, XP or Vista with DirectX 9 and Windows Media Player 9 or later. The software is free and is available on www.yawcam.com.

The advantage of this software is that it is very easy to use and it requires only the PC and web camera. However, the PC to which the web camera is attached should be continuously ON for 24 hours. Instead of web camera, IP (Internet Protocol) camera can be used.

\section{Results and Discussions}

The proposed systems are tested on the model of smart home (which is shown in Figure 1). The web camera based security system detects the motion and sends email to the home owner. The system is very simple and easy to use. There are various parameters which can be adjusted in this software. Streaming of videos is also possible with this software.

The developed GSM based security system gives good response to the sensor and sends SMS when it detects the fire or temperature is increased above desired level or detection of intrusion at the windows. The time taken by the system to deliver the SMS is dependent on the coverage area or range of the specified mobile network. If the mobile is in the range of the system then the SMS is delivered in 25-30 seconds.

Advantages of the proposed system:

1. As the system is SMS based, there is no need to have extra circuitry to transmit SMS. Mobile networks are used for transmission.

2. It is very cost effective, as day by day the cost of SMS is reducing.

Drawbacks of the proposed system:

1. All over the world, there could be a area where the mobile network is not established, so no connectivity of mobile phones in that area. Therefore, SMS cannot be delivered.

2. Older people still are not familiar with the use of mobile and find it difficult to see the SMS on mobile.

\section{Conclusions}

The GSM based home security system has been designed and tested with the mobile network. The user can get alerts anywhere through the GSM technology thus making the system location independent. A flexible way to control and explore the services of the mobile, AT commands is used in the system. The communication of home is only through the SMS which has been tested with the mobile networks and is working on any mobile network.

The web camera based security system is very easy, user friendly and software has many features. It will be more easy to use IP camera instead of web camera. However, the cost of IP camera is more. Similar softwares are available on internet which will perform the same task. This type of system is useful when the owner is out of station and the home is locked. By installing the web camera at the door site, intruder can be detected and owner can receive a 
mail telling the intruder entry in a home. If the nearby police station email id is also configured in the system, then the intrusion mail can be received by police also and necessary action can be taken.

The system has tested on the model of smart home and further it will be tested in actual home. The complexity of the algorithm of the system can be increased by introducing number of sensors to make the energy efficient home.

\section{Acknowledgements}

I would like to thank to Mr. THOKALE JITIN ISHWAR, the student of M.Sc. Part-II of Department of Electronic Science, University of Pune who helped a lot to complete this research work.

\section{References}

[1] The-History-of-Home-Security 4th July 2010 [Online]. Available: http://ezinearticles.com.

[2] V. Karri and J. S. Daniel Lim, "Method and Device to Communicate via SMS After a Security Intrusion”, 1st International Confe-rence on Sensing Technology, Palmerston North, New Zealand, (2005) November 2123.

[3] Y. Zhao and Z. Ye, “Low cost GSM/GPRS BASED wireless home security system”, IEEE Trans. Consumer Electron, vol. 56, no. 4, (2007) January, pp. 546-567.

[4] Z. Bing, G. Yunhung, L. Bo, Z. Guangwei and T. Tian, "Home Video Security Surveillance”, Info-Tech and Infonet, 2001,Proceedings,ICII 2001-Beijing. 2001 International Conference, vol. 3, pp. 202-208.

[5] M. Meyer, M. Hotter and T. Ohmacht, "A new system for Video-based Detection of moving objects and its integration into digital networks”, Security Technology 1996, 30th Annual 1996 International Carnahan Conference, (1996), pp. 105-110.

[6] Mae , Y,; Sasao , N .; INNoue ,K. ; Arai,T.; “Person Detection by Mobile Manipulator for Monitoring”,SICE 2003 Annual Conference, pages-2801-2806.

[7] “SMS Based Wireless Global Range Automation \& Security System”, Sudipan Saha, Sutasom Bhaumik.

[8] “Analysis and Performance of a Low Cost SMS Based Home Security System”, Sheikh Izzal Azid, Sushil Kumar, International Journal of Smart Home, vol. 5, no. 3, (2011) July.

[9] M. Butt, M. Khanam, A. Khan, M. Sikandar and H. Khiyal, "Controlling Home Appliances Remotely Through Voice Command”, (IJACSA) International Journal of Ad-vanced Computer Science and Applications, Special Issue on Wire-less \& Mobile Networks, pp. 35-39.

\section{Authors}

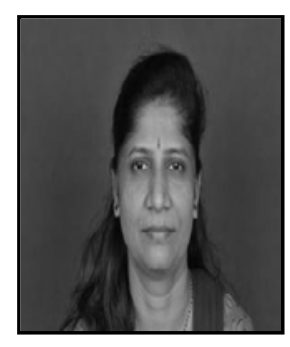

\section{Jayashri A. Bangali}

Kaveri College of Science and Commerce, Pune, India, e-mail: jayashri789@yahoo.com

J. A. Bangali born in 1974, is Asst. Prof. in the Department of Electronics, Kaveri College of Science and Commerce, Erandwane, Pune, India. She received her M. Sc. in 1996 and M.Phil. in 2009 from University of Pune, India. She has registered for Ph. D. in June 2010 under the guidance of Dr.A.D.Shaligarm in Pune University, India. She has around 20 publications in the National and International Journals/Conferences. Her research interest includes smart space automation, lighting research, PC/Micro controller based Instrumentation and sensors. 


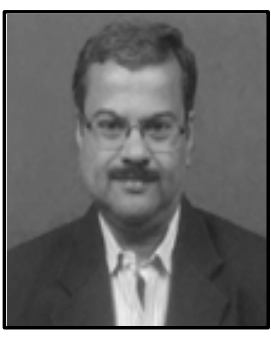

Dr. Arvind D. Shaligram

Department of Electronic Science, University of Pune, India

A. D. Shaligram born in 1960, is professor and Head of the Department of Electronic Science, University of Pune, Pune, India. He received his M. Sc. in 1981 and Ph. D. in 1986 from the same University. He has more than 344 papers out of which 100 papers are in International/National Journals, 21 invited talks and rest in conference proceedings. He has guided 23 students for Ph.D.,15 for M.Phil. and over 150 students for their M.Sc. thesis. He has completed 22 research projects funded by various funding agencies in India as Principal or co-investigator. His research interest includes fiber optic and optical waveguide sensors, PC/Micro controller based Instrumentation and Biomedical Instrumentation and sensors, Embedded systems, Nanoelectronics and Wireless Sensor Network. 